

Luiza Larangeira da Silva Mello

Natureza e Artifício

Sérgio Buarque de Holanda e as formae mentis portuguesa e espanhola na conquista e colonização do Novo Mundo

Dissertação de Mestrado

Dissertação apresentada como requisito parcial para obtenção do título de Mestre pelo Programa de PósGraduação em História da PUC-Rio.

Orientador: Prof. Ricardo Augusto Benzaquen de Araújo 


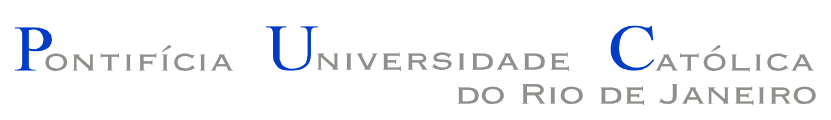

Luiza Larangeira da Silva Mello

\section{Natureza e Artifício \\ Sérgio Buarque de Holanda e as formae mentis portuguesa e espanhola na conquista e colonização do Novo Mundo}

Dissertação apresentada como requisito parcial para obtenção do título de Mestre pelo Programa de PósGraduação em História da PUC-Rio. Aprovada pela Comissão Examinadora abaixo assinada.

\section{Profo Ricardo Augusto Benzaquen de Araujo} Orientador

Departamento de História-PUC-Rio

Prof ${ }^{\circ}$ Robert Wegner Departamento de Pesquisa FIOCRUZ

Profo IImar Rohloff de Mattos Departamento de História - PUC-Rio

Profo João Pontes Nogueira Vice-Decano de Pós-Graduação do Centro de Ciências Sociais PUC-Rio

Rio de Janeiro, 12 de setembro de 2005 
Todos os direitos reservados. É proibida a reprodução total ou parcial do trabalho sem autorização da universidade, da autora e do orientador.

\section{Luiza Larangeira da Silva Mello}

Graduou-se em História na Pontifícia Universidade Católica do Rio de Janeiro (PUC-Rio) em 2002. Participou do Projeto Integrado de Pesquisa Os Modernos Descobridores do Brasil, entre 1998 e 2003.

Ficha Catalográfica

Mello, Luiza Larangeira da Silva

Natureza e artifício: Sérgio Buarque de Holanda e as formae mentis portuguesa e espanhola na conquista e colonização do Novo Mundo / Luiza Larangeira da Silva Mello ; orientador: Ricardo Augusto Benzaquen de Araújo. Rio de Janeiro : PUC-Rio, Departamento de História, 2006.

86 f. ; $30 \mathrm{~cm}$

Dissertação (mestrado) - Pontifícia Universidade Católica do Rio de Janeiro, Departamento de História.

Inclui bibliografia.

1. História - Teses. 2. Holanda, Sérgio Buarque de. 3. Historiografia brasileira. 4. Brasil colonial. 5. Cultura do renascimento. I. Araújo, Ricardo A. Benzaquen de. II. Pontifícia Universidade Católica do Rio de Janeiro. Departamento de História. III. Título. 


\section{Agradecimentos}

Dedico meus sinceros agradecimentos a Ricardo Benzaquen de Araújo, pela inteligência, carinho e paciência na orientação desta dissertação; a Robert Wegner, da banca examinadora, pelo apoio, ajuda e sugestões; a Ilmar Rohloff de Mattos, também da banca examinadora, pelo incentivo e sugestões; ao Departamento de História da PUC-Rio e a Edna Maria Lima Timbó; à PUC-Rio e ao CNPq; a Isis Dietrich Larangeira, pelo apoio, paciência e ajuda no penoso trabalho de revisão e formatação desta dissertação; a Carolina Miceli de Araújo, pela amizade, conversas e traduções; a Karina Vasquez, pela amizade e sugestões; a Luiz Alencar Reis da Silva Mello e Manoel Egrejas, pelo apoio, carinho e confiança; e a Fernanda Brandão de Araújo, pelo carinho, amizade e constante incentivo. 


\section{Resumo}

Mello, Luiza Larangeira da Silva; Araújo, Ricardo Augusto Benzaquen de. Natureza e Artifício. Sérgio Buarque de Holanda es as formae mentis portuguesa e espanhola na conquista e colonização do Novo Mundo. Rio de Janeiro, 2005. 86p. Dissertação de Mestrado - Departamento de História, Pontifícia Universidade Católica do Rio de Janeiro.

Em Visão do Paraíso, Sérgio Buarque de Holanda compara as formae mentis portuguesa e espanhola, na época da conquista e colonização do Novo Mundo. Estas formae mentis podem ser relacionadas a distintas modalidades de crença e a diferentes tipos de racionalidade que caracterizaram as mais diversas manifestações da subjetividade renascentista. Ambos os colonizadores, em seu primeiro contato com as terras descobertas, acreditaram poder encontrar nelas o Paraíso Terrestre e os motivos a ele associados, descritos nos textos clássicos e medievais. Os portugueses, plásticos e realistas, depuraram o mito dos elementos mais fantásticos e sobrenaturais, mantidos nas versões espanholas dos motivos edênicos. O argumento central da dissertação se desenvolve em torno da idéia de que as diferenças e semelhanças entres as formae mentis de portugueses e espanhóis estão relacionadas à ambigüidade dos elementos arcaicos e modernos que marcaram a cultura do Renascimento.

\section{Palavras-chave}

Sérgio Buarque de Holanda; Historiografia brasileira; Brasil colonial; Cultura do Renascimento. 


\section{Abstract}

Silva Mello, Luiza Larangeira da; Araújo, Ricardo Augusto Benzaquen de. Nature and Art. Sérgio Buarque de Holanda and the Portuguese and Spanish's formae mentis in the New World's discovery and colonization. Rio de Janeiro, 2005. 86p. MSc Dissertation - Departamento de História, Pontifícia Universidade Católica do Rio de Janeiro.

In Visão do Paraíso, Sérgio Buarque de Holanda compares the Portuguese forma mentis with the Spanish's, during the time of the discovery and colonisation of the New World. These formae mentis can be related to the distinct modes of belief and the different kinds of rationalism that distinguished the various expressions of the Renaissance self. Both the Portuguese and the Spanish, in their early contact with the new lands, believed they would find the Earthly Paradise described in the classic and medieval texts. The Portuguese, who were more plastic and realistic, removed the fantastic and supernatural traits from the myth. The Spanish, on the other hand, not only kept but also intensified these traits. The dissertation's main discussion is about the idea that the differences and the similarities between the Portuguese and the Spanish's formae mentis are related with the ambiguity present in the archaic and modern traits that characterize Renaissance culture.

\section{Keywords}

Sérgio Buarque de Holanda; Brazilian historiography; Colonial Brazil; Renaissance culture. 


\section{Sumário}

1 Introdução $\quad 9$

2 O "outro Brasil" 17

2.1. Plasticidade e Realismo 17

2.2. Adaptação radical 23

2.3. Plasticidade e Idealismo 27

2.4. Plasticidade e Paradoxo 33

2.5. O "outro Peru" 40

3 Através e apesar da natureza 42

3.1. As ambigüidades aparentes 42

3.2. O Barroco: mito, razão e lábia 51

3.3. O Éden interior e o Éden exterior 53

4 A flexibilidade tática $\quad 61$

4.1. As ambigüidades essenciais $\quad 61$

4.2. A plasticidade empática $\quad 62$

$\begin{array}{ll}\text { 4.3. Natureza e Artifício } & 73\end{array}$

5 Conclusão $\quad 82$

6 Referências bibliográficas $\quad 84$ 
Foi dessa capacidade de certas aspirações de fundo irracional que tantos teóricos e demagogos de nossos dias puderam, notoriamente, deduzir a importância do mito na vida dos povos. Pois o mito é o meio mais fecundo de se submeterem as gentes a uma dieta rigorosa, que encaminha os seus intentos e as suas vontades a certos fins magnificos, embora só obscuramente suspeitados. E, por outro lado, nos momentos em que se racionalizam as confusas aspirações é quando, justamente, costumam repontar certas razões contrárias, hesitações, ponderações amolecedoras de toda vontade e disciplina.

Sérgio Buarque de Holanda, Tentativas de Mitologia 